\title{
Synthesis of Poly(3,4,5-trihydroxybenzoate) dendrimers from Polyphenols and Their Chemiluminescence
}

\author{
Dai-Il Jung, ${ }^{*}$ Ju-Hyun Song, Eun-Hye Shin, Yun-Young Kim, Do-Hun Lee, Soon-Kyu Choi, and Jung-Tai Hahn ${ }^{\dagger}$ \\ Department of Chemistry, Dong-A University, Busan 604-714, Korea. *E-mail: dijung@dau.ac.kr \\ ${ }^{\dagger}$ Department of Beautycare, Young-Dong University, Chungbuk 370-701, Korea \\ Received October 23, 2009, Accepted December 29, 2009
}

Key Words: Chemiluminescence, Polyphenols, Gallic acid, (3,4,5-Trihydroxybenzoate ester) dendrimers, Symmetry

Dendrimer chemistry is rapidly expanding both for fundamental reasons as well as due to requirements in technological applications. ${ }^{1}$ A recent interesting development in dendrimer chemistry concerns the coordination of metal ions by interior branches or exterior units. ${ }^{2-6}$

Dendrimers containing photoactive units ${ }^{7-17}$ are particularly interesting for two reasons: (1) cooperation among the photoactive components can allow the dendrimer to perform specific functions, and (2) changes in the properties of photoactive components can be exploited to monitor the participation of dendrimers in chemical processes.

Polyphenols such as pyrogallol and gallic acid (GA) produce a singlet oxygen in the presence of hydrogen peroxide in an alkaline medium and emit light. GA has also been utilized for selective $\mathrm{Co}^{2+}$ detection using a chemiluminescence (CL) flow injection system. The CL intensities of polyphenols are considerably weaker than those of luminal and acridinium ester, which are representative CL compounds. Hence, trans-4-(3propionic acid)phenylboronic acid and 4-biphenylboronic acid in the presence of peroxidase or 1-ethyl-3-(3-dimethylaminopropyl)carbodiimide have been used as an enhancer for highly sensitive polyphenol CL determination. However, methods to derive polyphenols with strong CL intensity have not been reported yet. $^{18}$

In recent times, after a new polymer, poly(amidoamine) dendrimer, was synthesized, dendrimers have been applied to material engineering based on optics in nanoscale science. The poly(propylene amine) family that was functionalized in the periphery with fluorescent dansyl units interacts with $\mathrm{Co}^{2+}$. More recently, acridinium dendrimers have been conjugated with bovine serum albumin, and its CL was estimated to be six times that of the acridinium salt.

In this paper, we report the design of new types of polyphenol dendrimers having an increased number of chemiluminophores for high CL intensity; they also have hydroxyl groups in their periphery to form hydrogen bonds with an analyst. Polyphenol dendrimers having GA in the periphery were synthesized, and their CL was measured. We synthesized first-generation polyphenol dendrimers with GA units in the periphery by employing a divergent method using phenol, pyrocatechol, resorcinol, hydroquinone, 1,2,4-trihydroxybenzene, and 3,4,5-trihydroxybenzene as the core molecules (Schemes 1-3).

The esterification of 3,4,5-trihydroxybenzoyl chloride with phenol yielded 2a in $80 \%$. After the debenzylation of $2 \mathbf{a}$, we were able to separate $\mathbf{3 a}$ and the byproduct by chromatographic purification. Therefore, the desired zero-generation ester 3a was not isolated. Compounds $4 \mathbf{a}$ and $\mathbf{5 a}$ were synthesized by repeating this esterification and debenzylation, respectively, but the yield of the first-generation ester 5a was 91\%. The esterification of 3,4,5-trihydroxybenzoyl chloride with pyrocatechol, 3,4,5-trihydroxybenzoyl chloride with resorcinol, and 3,4,5trihydroxybenzoyl chloride with hydroquinone yielded $\mathbf{2 b}$ (95\%), 2c (96\%), and 2d (91\%), respectively. The zero-generation esters $\mathbf{3 b}, \mathbf{3 c}$, and $\mathbf{3 d}$ and first-generation esters $\mathbf{5 b}, \mathbf{5 c}$, and $\mathbf{5 d}$ were synthesized as described above. The yields of the polyphenol dendrimers decreased with increasing dendrimer generation. Compounds $\mathbf{4 a}, \mathbf{4 b}, \mathbf{4 c}$, and $\mathbf{4 d}$ were debenzylated in high yield by catalytic reduction under hydrogen gas in the presence of palladium black at ambient temperature. Benzylation must be optimal for protecting the phenols. The esterification of 3,4,5-trihydroxybenzoyl chloride with 1,3,5-trihydroxybenzene and 3,4,5-trihydroxybenzoyl chloride with 1,2,4-trihydroxybenzene as an asymmetric polyol yielded $4 \mathbf{e}$ $(69 \%)$ and $\mathbf{4 f}(42 \%)$, respectively. The zero-generation esters $\mathbf{3 e}$ and $\mathbf{3 f}$ and first-generation esters $\mathbf{5 e}$ and $\mathbf{5 f}$ were synthesized as described above. The mass spectra of the zero-generation esters 3a, 3b, 3c, 3d, 3e, and 3f and first-generation esters 5a, $\mathbf{5 b}, \mathbf{5 c}, \mathbf{5 d}, \mathbf{5 e}$, and $\mathbf{5 f}$ were obtained by matrix-assisted laser desorption/ionization-time-of-flight mass spectrometry (MALDI TOF MS) using 2,5-dihydroxybenzoic acid as the matrix. The first-generation esters $\mathbf{5 a}, \mathbf{5 b}, \mathbf{5 c}, \mathbf{5 d}, \mathbf{5 e}$, and $\mathbf{5 f}$ were very stable in $\mathrm{MeOH}$. Compounds $\mathbf{5 a}, \mathbf{5 b}, \mathbf{5 e}$, and $\mathbf{5 f}$ showed maximum CL intensities when $100 \mathrm{mM}$ sodium hydroxide and 500 $\mathrm{mM}$ hydrogen peroxide were added. Both the intensities of the maximum light emission and the length of the CL period of $\mathbf{5 a}, \mathbf{5 b}, \mathbf{5 e}$, and $\mathbf{5 f}$ were more than that of GA. The time of maximum light emission of $\mathbf{5 a}, \mathbf{5 b}, \mathbf{5 e}$, and $\mathbf{5 f}$ were 50 and $60 \mathrm{~s}$ after the injection of hydrogen peroxide. The CL intensities 5 min after the injection of hydrogen peroxide were approximately half the maximum CL intensities. Such polyphenol compounds, which emit light for such a long CL life, have not been found. The CL intensities of $\mathbf{5 b}$ and $\mathbf{5 e}$ in $\mathrm{MeOH}$ were approximately 400- and 600-fold stronger than that of GA, respectively (Table 1). ${ }^{19}$

The CL intensities per GA unit of $\mathbf{5 b}$ and $\mathbf{5 e}$ were approximately equal, which suggests that increasing the number of 

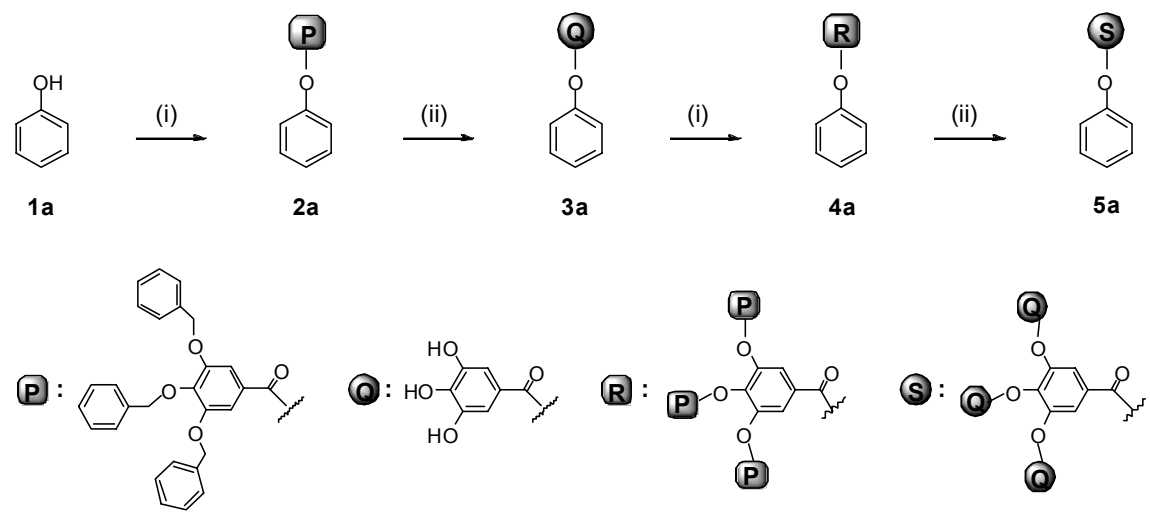

Scheme 1. Synthetic route of 2a-5a. Reagents and conditions: (i) $\mathrm{CHCl}_{3}$, pyridine, 4-dimethylaminopyridine, and 3,4,5-tribenzyloxybenzoyl chloride (ii) Pd-Black, $\mathrm{H}_{2}$, and $\mathrm{CHCl}_{3}-\mathrm{MeOH}$

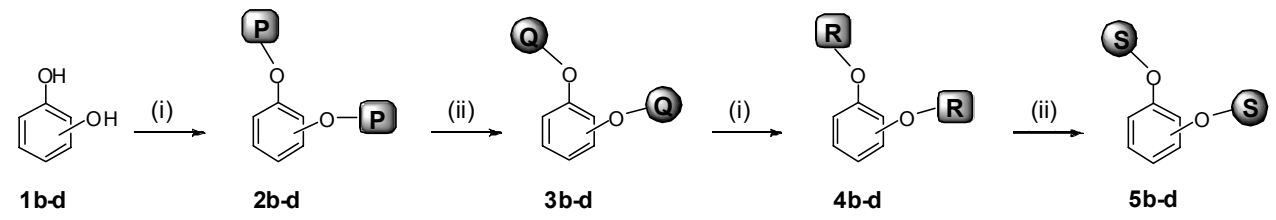

Scheme 2. Synthetic route of $\mathbf{2 b}, \mathbf{c}, \mathbf{d}-\mathbf{5} \mathbf{b}, \mathbf{c}, \mathbf{d}$. Reagents and conditions: (i) $\mathrm{CHCl}_{3}$, pyridine, 4-dimethylaminopyridine, and 3,4,5-tribenzyloxybenzoyl chloride (ii) Pd-Black, $\mathrm{H}_{2}$, and $\mathrm{CHCl}_{3}-\mathrm{MeOH}$<smiles>Oc1cc(O)cc(Oc2ccccc2)c1</smiles>

$1 e$<smiles>CC#CCOc1ccc(Oc2ccccc2)c(OCC)c1</smiles><smiles>[O-]c1cc(O[Hg])cc(O[Hg])c1</smiles>

3e

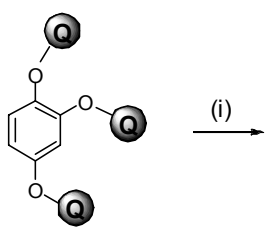

$3 f$<smiles>CCOc1cc(O[Ga])cc(Oc2ccccc2)c1</smiles>

$4 \mathrm{e}$<smiles>[O-]c1cc(O[Hg])cc(O[Hg])c1</smiles>

$5 e$

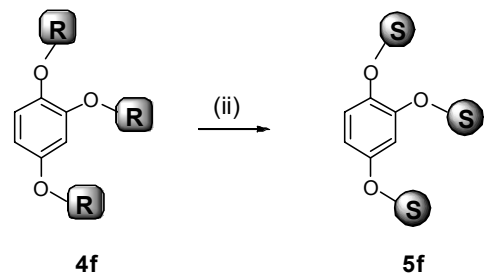

Scheme 3. Synthetic route of 2e,f-5e,f. Reagents and conditions: (i) $\mathrm{CHCl}_{3}$, pyridine, 4-dimethylaminopyridine, and 3,4,5-tribenzyloxybenzoyl chloride (ii) $\mathrm{Pd}-\mathrm{Black}, \mathrm{H}_{2}$, and $\mathrm{CHCl}_{3}-\mathrm{MeOH}$

Table 1. Relative CL intensities of GA, $\mathbf{5 a}, \mathbf{5 b}, \mathbf{5 e}$, and $\mathbf{5 f}$ in $\mathrm{MeOH}$

\begin{tabular}{|c|c|c|c|c|c|}
\hline Core & Compound $^{a}$ & $\mathrm{NaOH}(\mathrm{mM})$ & $\mathrm{H}_{2} \mathrm{O}_{2}(\mathrm{mM})$ & Integral photon count $\left(\times 10^{4}\right)$ & Relative CL intensity $^{b}$ \\
\hline & GA & 100 & 500 & 4.8 & 1.0 \\
\hline Phenol & 5a & 100 & 500 & 977.8 & 203.7 \\
\hline 1,2-dihydroxybenzene & $5 \mathbf{b}$ & 100 & 500 & 2025.0 & 421.0 \\
\hline 1,3,5-dihydroxybenzene ${ }^{c}$ & $5 e$ & 100 & 500 & 2986.0 & 622.0 \\
\hline 1,2,4-dihydroxybenzene ${ }^{d}$ & $5 f$ & 100 & 500 & 123.1 & 25.6 \\
\hline
\end{tabular}

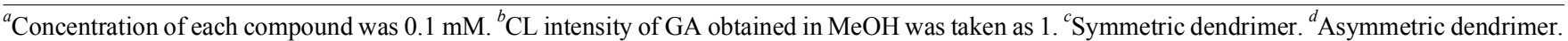

chemiluminophores was effective in increasing the CL intensity of polyphenol dendrimers. The increase in the CL intensity of GA was accomplished by the formation of a dendric structure. However, the CL intensity of $\mathbf{5 f}$ as an asymmetric dendrimer in $\mathrm{MeOH}$ was weaker than that of $\mathbf{5 e}$, and it is suggested that the symmetry of chemiluminophores was effective in increasing the CL intensity of polyphenol dendrimers.

In conclusion, polyphenol dendrimers were synthesized to obtain a strong CL compound, and their CL intensities were found to be considerably stronger than the CL intensity of GA. 
The esterification of the hydroxyl groups of GA in the dendrimer was very effective in developing a strong CL. Further, the relationship between the CL intensity and structure of polyphenol dendrimers must be clarified to understand the reason behind the strong light emission of high-per-branch compounds such as poly(3,4,5-trihydroxybenzoate ester) dendrimers. Polyphenol CL dendrimers can be used for a wide variety of CL assays by utilizing the hydroxyl groups of the polyphenol for forming a hydrogen bond with oxygen in the analyte structure.

\section{Experimental Section}

Melting points were determined using an electrothermal capillary melting point apparatus and uncorrected. Thin layer chromatography (TLC) was performed on glass plates coated with silicon oxide (silica gel $60 \mathrm{~F}_{254}$ ), and compounds were visualized using a UV lamp. ${ }^{1} \mathrm{H}$ and ${ }^{13} \mathrm{C}$ NMR spectra were obtained with Bruker AC $2000(200 \mathrm{MHz})$ and Varian Gemini (200 or $300 \mathrm{MHz}$ ) spectrometers. Mass spectra were measured with HP $5890 \mathrm{GC} /$ Mass $(70 \mathrm{eV}, \mathrm{EI})$. The organic solvents and chemicals were obtained from commercial products and purified by the appropriate methods before use. Except where explicitly stated, all the starting materials were purchased from Aldrich, Fluka, Fisher, Lancaster, or TCI chemical companies and used as received.

General procedure for the synthesis of 2-5. To a stirred solution of 4-dimethylaminopyridine (DMAP, $5 \mathrm{mmol}$ ), phenol (or polyphenol) 1 ( $5 \mathrm{mmol})$, and pyridine $(0.7 \mathrm{~mL})$ in $\mathrm{CHCl}_{3}(60$ $\mathrm{mL}$ ) was added 3,4,5-tribenzyloxybenzoyl chloride (5 mmol). After the mixture was refluxed under $\mathrm{N}_{2}$ for $5 \mathrm{~h}$, the organic layer was filtered, concentrated, and dried. A white solid product 2 was obtained by flash column chromatography on silica gel $\left(\mathrm{CH}_{2} \mathrm{Cl}_{2}\right)$. To obtain 3, a mixture of synthesized $2(3.25 \mathrm{mmol})$ and activated carbon $(0.15 \mathrm{~g})$ in $\mathrm{CHCl}_{3}(24 \mathrm{~mL})$ and $\mathrm{MeOH}$ $(12 \mathrm{~mL})$ was stirred under hydrogen gas for $3 \mathrm{~h}$ at room temperature. After the reaction mixture was stirred for $3 \mathrm{~h}$, the organic layer was filtered, concentrated, and dried. A light yellow solid product was then collected. After the debenzylation of $\mathbf{2}$, we were not able to separate $\mathbf{3}$ and the byproduct by chromatographic purification. Therefore, the desired zero-generation ester $\mathbf{3}$ was not isolated. Compounds $\mathbf{4}$ and $\mathbf{5}$ were synthesized in high yield by repeating this esterification and debenzylation. The synthesis of polyphenol dendrimers by using the corresponding polyphenol was attempted using similar methods.

Phenyl 3,4,5-tris(benzyloxy)benzoate (2a): Yield: $80 \%$; mp 122 - $123{ }^{\circ} \mathrm{C} ;{ }^{1} \mathrm{H}$ NMR $\left(200 \mathrm{MHz}, \mathrm{CDCl}_{3}\right) \delta 7.40(\mathrm{~m}, 22 \mathrm{H}), 5.20$ $(\mathrm{s}, 6 \mathrm{H}) ;{ }^{13} \mathrm{CNMR}\left(50 \mathrm{MHz}, \mathrm{CDCl}_{3}\right) \delta 164.7,152.6,150.9,137.3$, 136.5, 129.4, 128.5, 128.2, 128.1, 127.5, 125.8, 124.4, 123.66, 109.6, 109.4, 75.3, 71.3, 71.2; Anal. Calcd. for $\mathrm{C}_{34} \mathrm{H}_{28} \mathrm{O}_{5}$ : C, 79.05; H, 5.46. Found: C, 79.08; H, 5.42.

5-(Phenoxycarbonyl)benzene-1,2,3-triyl tris(3,4,5-tris(benzyloxy)benzoate) (4a): Yield: $84 \%$; mp $82-83{ }^{\circ} \mathrm{C} ;{ }^{1} \mathrm{H}$ NMR $\left(200 \mathrm{MHz} \mathrm{CDCl}_{3}\right) \delta 7.75(\mathrm{~m}, 58 \mathrm{H}), 4.98(\mathrm{~m}, 18 \mathrm{H}) ;{ }^{13} \mathrm{C} \mathrm{NMR}$ $\left(50 \mathrm{MHz}, \mathrm{CDCl}_{3}\right) \delta 163.3,152.7,152.7,150.7,144.2,143.4$, $137.2,136.2,135.9,128.1,127.9,127.7,127.4,126.0,123.2$, 123.0, 122.7, 122.5, 109.8, 109.4, 75.4, 75.1, 74.8, 71.4, 71.2, 70.9; Anal. Calcd. for $\mathrm{C}_{97} \mathrm{H}_{76} \mathrm{O}_{17}$ : C, 76.97; H, 5.06. Found: $\mathrm{C}$, 76.91; H, 5.09.
5-(Phenoxycarbonyl)benzene-1,2,3-triyl tris-(3,4,5-trihydroxybenzoate) (5a): Yield: 91\%; mp $202-204{ }^{\circ} \mathrm{C} ;{ }^{1} \mathrm{H}$ NMR (200 MHz, DMSO) $\delta 9.36(\mathrm{~s}, 3 \mathrm{H}), 9.30(\mathrm{~s}, 3 \mathrm{H}), 9.19(\mathrm{~s}, 3 \mathrm{H})$, $7.48(\mathrm{~m}, 13 \mathrm{H}) ;{ }^{1} \mathrm{H}$ NMR $\left(200 \mathrm{MHz}, \mathrm{DMSO}+\mathrm{D}_{2} \mathrm{O}\right) \delta 7.55(\mathrm{~m}$, $13 \mathrm{H}) ;{ }^{13} \mathrm{C} \mathrm{NMR}(125 \mathrm{MHz}, \mathrm{DMSO}) \delta 163.5,163.4,162.4,150.6$, 145.8, 145.7, 140.4, 139.9, 129.7, 127.1, 126.3, 122.7, 118.9, 116.9, 116.2, 115.3, 109.3, 109.2; MALDI-TOF MS 702.54 $[\mathrm{M}+\mathrm{Na}]^{+}$; Anal. Calcd. for $\mathrm{C}_{34} \mathrm{H}_{22} \mathrm{O}_{17}$ : C, 58.13; H, 3.16. Found: C, 58.11; H, 3.20.

1,2-Phenylene bis(3,4,5-tris(benzyloxy)benzoate) (2b): Yield: $92 \%$; mp $122-123{ }^{\circ} \mathrm{C} ;{ }^{1} \mathrm{H}$ NMR $\left(200 \mathrm{MHz}, \mathrm{CDCl}_{3}\right) \delta 7.34$ $(\mathrm{m}, 38 \mathrm{H}), 5.00(\mathrm{~s}, 4 \mathrm{H}), 4.92(\mathrm{~s}, 8 \mathrm{H}) ;{ }^{13} \mathrm{C} \mathrm{NMR}\left(50 \mathrm{MHz}, \mathrm{CDCl}_{3}\right)$ $\delta 163.7,152.6,143.1,142.5,137.3,136.2,128.5,128.2,128.1$, 128.0, 127.8, 127.6, 125.7, 123.6, 109.4, 109.3, 75.2, 75.0, 74.9, 71.1, 71.0, 70.9; Anal. Calcd. for $\mathrm{C}_{62} \mathrm{H}_{50} \mathrm{O}_{10}$ : C, 77.97; H, 5.28. Found: C, 77.94; H, 5.22.

1,2-Di-5-(phenoxycarbonyl)benzene-1,2,3-triyl tris(3,4,5tris(benzyloxy)benzoate) (4b): Yield: $47 \%$; mp $157-159{ }^{\circ} \mathrm{C}$; ${ }^{1} \mathrm{H}$ NMR $\left(200 \mathrm{MHz}, \mathrm{CDCl}_{3}\right) \delta 7.35(\mathrm{~m}, 110 \mathrm{H}), 5.19(\mathrm{~s}, 12 \mathrm{H})$, $5.13(\mathrm{~s}, 24 \mathrm{H}) ;{ }^{13} \mathrm{C} \mathrm{NMR}\left(50 \mathrm{MHz}, \mathrm{CDCl}_{3}\right) \delta 162.1,152.7,136.2$, 128.6, 128.2, 128.1, 127.5, 123.4, 110.1, 110.0, 75.2, 71.5, 71.5, 71.2; Anal. Calcd. for $\mathrm{C}_{188} \mathrm{H}_{146} \mathrm{O}_{34}$ : C, 76.56; H, 4.99. Found: C, 76.53; H, 4.92.

1,2-Di-5-(phenoxycarbonyl)benzene-1,2,3-triyl tris-(3,4,5trihydroxybenzoate) (5b): Yield: $47 \%$; mp $180-182{ }^{\circ} \mathrm{C} ;{ }^{1} \mathrm{H}$ NMR (200 MHz, DMSO) $\delta 9.33$ (s, 12H), 9.19 (s, 6H), 7.48 (m, 20H); ${ }^{1} \mathrm{H}$ NMR $\left(200 \mathrm{MHz}, \mathrm{DMSO}+\mathrm{D}_{2} \mathrm{O}\right) \delta 7.55$ (m, 20H); ${ }^{13} \mathrm{C}$ NMR (125 MHz, DMSO) $\delta 163.5,163.5,162.4,150.6$, $145.8,145.7,140.4,139.9,129.7,127.1,126.3,122.7,118.9$, 116.9, 116.2, 115.3, 109.3, 109.2; MALDI-TOF MS 1350.27 $[\mathrm{M}+\mathrm{Na}]^{+}$; Anal. Calcd. for $\mathrm{C}_{62} \mathrm{H}_{38} \mathrm{O}_{34}: \mathrm{C}, 56.12 ; \mathrm{H}, 2.89$. Found: C, 56.17; H, 2.85.

1,3-Phenylene bis(3,4,5-tris(benzyloxy)benzoate) (2c): Yield: $96 \%$; mp 93 - $94{ }^{\circ} \mathrm{C} ;{ }^{1} \mathrm{H}$ NMR $\left(200 \mathrm{MHz}, \mathrm{CDCl}_{3}\right) \delta 7.54$ $(\mathrm{s}, 4 \mathrm{H}), 7.31(\mathrm{~m}, 34 \mathrm{H}), 5.30(\mathrm{~s}, 4 \mathrm{H}), 5.18(\mathrm{~s}, 8 \mathrm{H}) ;{ }^{13} \mathrm{C} \mathrm{NMR}$ $\left(50 \mathrm{MHz}, \mathrm{CDCl}_{3}\right) \delta 164.4,152.6,151.4,137.3,136.5,129.9$, 128.5, 128.2, 128.1, 127.5, 124.7, 119.2, 115.9, 109.6, 109.5, 75.0, 71.3; Anal. Calcd. For $\mathrm{C}_{62} \mathrm{H}_{50} \mathrm{O}_{10}$ : C, 77.97; H, 5.28. Found: C, 77.99; H, 5.21 .

1,3-Di-5-(phenoxycarbonyl)benzene-1,2,3-triyl tris(3,4,5tris(benzyloxy)benzoate) (4c): Yield: $17 \%$; mp $113-114{ }^{\circ} \mathrm{C}$; ${ }^{1} \mathrm{H} \mathrm{NMR}\left(200 \mathrm{MHz}, \mathrm{CDCl}_{3}\right) \delta 7.36(\mathrm{~m}, 110 \mathrm{H}), 5.04(\mathrm{~m}, 36 \mathrm{H})$; ${ }^{13} \mathrm{C} \mathrm{NMR}\left(50 \mathrm{MHz}, \mathrm{CDCl}_{3}\right) \delta 163.7,152.7,152.5,137.3,136.5$, 128.2, 127.8, 127.5, 127.4, 124.3, 123.4, 122.7, 109.7, 109.3, 75.4, 74.8, 71.4, 71.1; Anal. Calcd. for $\mathrm{C}_{188} \mathrm{H}_{146} \mathrm{O}_{34}$ : C, 76.56; $\mathrm{H}$, 4.99. Found: C, 76.51; H, 5.03.

1,3-Di-5-(phenoxycarbonyl)benzene-1,2,3-triyl tris-(3,4,5trihydroxybenzoate) (5c): Yield: $64 \%$; mp $199-201{ }^{\circ} \mathrm{C} ;{ }^{1} \mathrm{H}$ NMR (200 MHz, DMSO) $\delta 9.11(\mathrm{~m}, 18 \mathrm{H}), 7.49(\mathrm{~m}, 20 \mathrm{H}) ;{ }^{1} \mathrm{H}$ NMR $\left(200 \mathrm{MHz}, \mathrm{DMSO}+\mathrm{D}_{2} \mathrm{O}\right) \delta 7.48(\mathrm{~m}, 20 \mathrm{H}) ;{ }^{13} \mathrm{C} \mathrm{NMR}$ (125 MHz, DMSO) $\delta 167.5,164.2,163.6,163.4,145.7,145.4$, 140.0, 137.9, 123.0, 122.7, 120.4, 118.0, 117.3, 116.9, 109.5, 109.3, 108.7; MALDI-TOF MS 1350.27 [M+Na $]^{+}$; Anal. Calcd. for $\mathrm{C}_{62} \mathrm{H}_{38} \mathrm{O}_{34}$ : C, 56.12; H, 2.89. Found: C, 56.09; H, 2.84.

1,4-Phenylene bis(3,4,5-tris(benzyloxy)benzoate) (2d): Yield: $91 \%$; mp $199-200{ }^{\circ} \mathrm{C} ;{ }^{1} \mathrm{H}$ NMR $\left(200 \mathrm{MHz}, \mathrm{CDCl}_{3}\right) \delta 7.55$ $(\mathrm{s}, 4 \mathrm{H}), 7.36(\mathrm{~m}, 34 \mathrm{H}), 5.19(\mathrm{~s}, 4 \mathrm{H}), 5.17(\mathrm{~s}, 8 \mathrm{H}) ;{ }^{13} \mathrm{C} \mathrm{NMR}(50$ $\left.\mathrm{MHz}, \mathrm{CDCl}_{3}\right) \delta 164.6,152.6,148.4,137.3,136.5,128.7,128.5$, 
128.3, 128.0, 127.8, 127.3, 124.2, 122.7, 122.6,109.8, 109.4, 75.2, 71.3; Anal. Calcd. for $\mathrm{C}_{62} \mathrm{H}_{50} \mathrm{O}_{10}$ : C, 77.97; H, 5.28. Found: C, 77.93; H, 5.32.

1,4-Di-5-(phenoxycarbonyl)benzene-1,2,3-triyl tris(3,4,5tris(benzyloxy)benzoate) (4d): Yield: 14\%; mp $211-213{ }^{\circ} \mathrm{C}$; ${ }^{1} \mathrm{H}$ NMR $\left(200 \mathrm{MHz}, \mathrm{CDCl}_{3}\right) \delta 7.34(\mathrm{~m}, 110 \mathrm{H}), 5.07$ (m, 36H); ${ }^{13} \mathrm{C} \mathrm{NMR}\left(50 \mathrm{MHz}, \mathrm{CDCl}_{3}\right) \delta 162.1,152.8,152.7,144.3,137.1$, 136.3, 128.3, 127.6, 127.3, 123.5, 123.3, 122.7, 110.3, 109.9, 109.4, 75.1, 71.4; Anal. Calcd. for $\mathrm{C}_{188} \mathrm{H}_{146} \mathrm{O}_{34}$ : C, 76.56; H, 4.99. Found: C, 76.53; H, 4.92 .

1,4-Di-5-(phenoxycarbonyl)benzene-1,2,3-triyl tris-(3,4,5trihydroxybenzoate) (5d): Yield: $23 \%$; mp $222-224{ }^{\circ} \mathrm{C} ;{ }^{1} \mathrm{H}$ NMR (200 MHz, DMSO) $\delta 9.30(\mathrm{~m}, 18 \mathrm{H}), 7.49(\mathrm{~m}, 20 \mathrm{H}) ;{ }^{1} \mathrm{H}$ $\mathrm{NMR}\left(200 \mathrm{MHz}, \mathrm{DMSO}+\mathrm{D}_{2} \mathrm{O}\right) \delta 7.47(\mathrm{~m}, 20 \mathrm{H}) ;{ }^{13} \mathrm{C} \mathrm{NMR}(125$ MHz, DMSO) $\delta 167.9,163.9,145.9,145.6,144.6,138.2,123.5$, 120.8, 118.5, 117.7, 117.4, 109.8, 109.2, 108.9; MALDI-TOF MS 1350.27 [M+Na $]^{+}$; Anal. Calcd. for $\mathrm{C}_{62} \mathrm{H}_{38} \mathrm{O}_{34}: \mathrm{C}, 56.12$; H, 2.89. Found: C, 56.15; H, 2.83.

Benzene-1,3,5-triyl tris(3,4,5-tris(benzyloxy)benzoate) (2e): Yield: 69\%; mp $196-200{ }^{\circ} \mathrm{C} ;{ }^{1} \mathrm{H}$ NMR $\left(200 \mathrm{MHz}, \mathrm{CDCl}_{3}\right) \delta$ $7.33(\mathrm{~m}, 54 \mathrm{H}), 5.17(\mathrm{~s}, 18 \mathrm{H}) ;{ }^{13} \mathrm{C} \mathrm{NMR}\left(50 \mathrm{MHz}, \mathrm{CDCl}_{3}\right) \delta$ $163.9,152.7,151.5,143.1,137.2,137.1,136.4,136.2,128.6$, 127.5, 123.8, 123.4, 113.4, 110.1, 109.5, 75.3, 74.9, 71.3, 71.1; Anal. Calcd. for $\mathrm{C}_{90} \mathrm{H}_{72} \mathrm{O}_{15}$ : C, 77.57; H, 5.21. Found: C, 77.53; H, 5.27 .

1,3,5-Tri-5-(phenoxycarbonyl)benzene-1,2,3-triyl tris(3,4,5tris(benzyloxy)benzoate) (4e): Yield: $25 \%$; mp $145-146{ }^{\circ} \mathrm{C}$; ${ }^{1} \mathrm{H}$ NMR (200 MHz, $\left.\mathrm{CDCl}_{3}\right) \delta 7.34(\mathrm{~m}, 162 \mathrm{H}), 5.08(\mathrm{~m}, 18 \mathrm{H})$; ${ }^{13} \mathrm{C} \mathrm{NMR}\left(50 \mathrm{MHz}, \mathrm{CDCl}_{3}\right) \delta 152.7,152.6,136.2,136.1,128.5$, 127.6, 123.4, 110.1, 109.5, 75.2, 71.3, 71.1; Anal. Calcd. for $\mathrm{C}_{188} \mathrm{H}_{146} \mathrm{O}_{34}$ : C, 76.56; H, 4.99. Found: C, 76.52; H, 5.04.

1,3,5-Tri-5-(phenoxycarbonyl)benzene-1,2,3-triyl tris-(3,4, 5-trihydroxybenzoate) (5e): Yield: $21 \%$; $\mathrm{mp} 238-239{ }^{\circ} \mathrm{C} ;{ }^{1} \mathrm{H}$ NMR (200 MHz, DMSO) $\delta 9.34$ (m, 18H), 9.17 (s, 9H), 7.48 $(\mathrm{m}, 27 \mathrm{H}) ;{ }^{1} \mathrm{H}$ NMR $\left(200 \mathrm{MHz}, \mathrm{DMSO}+\mathrm{D}_{2} \mathrm{O}\right) \delta 7.45(\mathrm{~m}, 27 \mathrm{H})$; ${ }^{13} \mathrm{C}$ NMR (125 MHz, DMSO) $\delta 163.9,145.9,144.7,139.9$, 118.6, 109.6; MALDI-TOF MS 1974.41 [M+Na] ; Anal. Calcd. for $\mathrm{C}_{62} \mathrm{H}_{38} \mathrm{O}_{34}$ : C, 56.12; H, 2.89. Found: C, 56.10; H, 2.93 .

Benzene-1,2,4-triyl tris(3,4,5-tris(benzyloxy)benzoate) (2f): Yield: $42 \%$; mp $149-150{ }^{\circ} \mathrm{C} ;{ }^{1} \mathrm{H}$ NMR $\left(200 \mathrm{MHz}, \mathrm{CDCl}_{3}\right) \delta$ $7.42(\mathrm{~m}, 54 \mathrm{H}), 5.19(\mathrm{~s}, 6 \mathrm{H}), 4.99(\mathrm{~s}, 6 \mathrm{H}), 4.93(\mathrm{~s}, 6 \mathrm{H}) ;{ }^{13} \mathrm{C} \mathrm{NMR}$ $\left(50 \mathrm{MHz}, \mathrm{CDCl}_{3}\right) \delta 152.7,136.2,128.6,127.6,123.8,119.8$, 117.5, 109.7, 109.5, 75.1, 71.3, 71.1; Anal. Calcd. for $\mathrm{C}_{90} \mathrm{H}_{72} \mathrm{O}_{15}$ : C, 77.57; H, 5.21. Found: C, 77.53; H, 5.26.

1,2,4-Tri-5-(phenoxycarbonyl)benzene-1,2,3-triyl tris $(3,4$, 5-tris(benzyloxy)benzoate) (4f): Yield: 7\%; mp 160 - $165^{\circ} \mathrm{C}$; ${ }^{1} \mathrm{HNMR}\left(200 \mathrm{MHz}, \mathrm{CDCl}_{3}\right) \delta 7.47(\mathrm{~m}, 162 \mathrm{H}), 5.15(\mathrm{~s}, 54 \mathrm{H}) ;{ }^{13} \mathrm{C}$ NMR $\left(50 \mathrm{MHz}, \mathrm{CDCl}_{3}\right) \delta 171.1,152.6,136.5,128.5,124.1$, 109.6, 75.1, 71.2; Anal. Calcd. for $\mathrm{C}_{188} \mathrm{H}_{146} \mathrm{O}_{34}$ : C, 76.56; H, 4.99. Found: C, 76.52; H, 4.93.

1,2,4-Tri-5-(phenoxycarbonyl)benzene-1,2,3-triyl tris-(3,4, 5-trihydroxybenzoate) (5f): Yield: $54 \%$; mp $234-235{ }^{\circ} \mathrm{C} ;{ }^{1} \mathrm{H}$ NMR (200 MHz, DMSO) $\delta 9.54$ (s, 18H), 9.41 (s, 9H), 7.01 (s, $27 \mathrm{H}) ;{ }^{1} \mathrm{H}$ NMR $\left(200 \mathrm{MHz}, \mathrm{DMSO}+\mathrm{D}_{2} \mathrm{O}\right) \delta 7.01(\mathrm{~s}, 27 \mathrm{H}) ;{ }^{13} \mathrm{C}$ NMR (125 MHz, DMSO) $\delta 167.5,162.7,145.9,145.4,140.4$, 137.9, 120.5, 117.5, 109.5, 109.3; MALDI-TOF MS 1974.32 $[\mathrm{M}+\mathrm{Na}]^{+}$; Anal. Calcd. for $\mathrm{C}_{62} \mathrm{H}_{38} \mathrm{O}_{34}: \mathrm{C}, 56.12 ; \mathrm{H}, 2.89$. Found: C, 56.08; H, 2.95.
Acknowledgments. This work was supported by a Dong-A University Research Fund (2008). This paper is dedicated to the late Dr. Chi-Sun Hahn to admire the high contributions of the academic world.

\section{References}

1. (a) Tomalia, D. A.; Durst, H. D. Top. Curr. Chem. 1993, 165, 193. (b) Fréchet, J. M. J. Science 1994, 263, 1710. (c) Ardoin, N.; Astruc, D. Bull. Soc. Chim. Fr. 1995, 132, 875. (d) Newkome, G. R.; Moorefield, C.; Vögtle, F. Dendritic Molecules: Concepts, Syntheses Perspectives; VCH: Weinheim, 1996. (e) Zeng, F.; Zimmerman, S. C. Chem. Rev. 1997, 97, 1681. (f) Venturi, M.; Serroni, S.; Juris, A.; Campagna, S.; Balzani, V. Top. Curr. Chem. 1998, 197, 193. (g) Gorman, C. Adv. Mater. 1998, 10, 295.

2. (a) Ottaviani, M. F.; Bosmann, S.; Turro, N. J.; Tomalia, D. A. J. Am. Chem. Soc. 1994, 116, 661. (b) Ottaviani, M. F.; Montalti, F.; Turro, N. J.; Tomalia, D. A. J. Phys. Chem. B 1997, 101, 158.

3. Zhao, M.; Sun, L.; Crooks, R. M. J. Am. Chem. Soc. 1998, 120, 4877.

4. Bosman, A. W.; Schenning, A. P. H. J.; Jansen, R. A. J.; Meijer, E. W. Chem. Ber. Recl. 1997, 130, 725.

5. Klein Gebbink, R. J. M.; Bosman, A. W.; Feiter, M. C.; Meijer, E. W.; Nolte, R. J. M. Chem. Eur. J. 1999, 5, 65.

6. Epperson, J. D.; Ming, L.-J.; Woosley, B. D.; Baker, G. R.; Newkome, G. R. Inorg. Chem. 1999, 38, 4498.

7. Campagna, S.; Denti, G.; Serroni, S.; Juris, A.; Venturi, M.; Ricevuto, V.; Balzani, V. Chem. Eur. J. 1995, 1, 211.

8. Bharathi, P.; Patel, U.; Kawaguchi, T.; Pessac, D. J.; Moore, J. S. Macromolecules 1995, 28, 5955.

9. Stewart, G. M.; Fox, M. A. J. Am. Chem. Soc. 1996, 118, 4354.

10. (a) Junge, D. M.; McGrath, D. V. Chem. Commun. 1997, 9, 857. (b) Junge, D. M.; McGrath, D. V.J. Am. Chem. Soc. 1999, 121, 4912.

11. (a) Issberner, J.; Vögtle, F.; De Cola, L.; Balzani, V. Chem. Eur. J. 1997, 3, 706. (b) Archut, A.; Vögtle, F.; De Cola, L.; Azzellini, G. C.; Balzani, V.; Ramanujam, P. S.; Berg, R. H. Chem. Eur. J. 1998, 4, 699. (c) Archut, A.; Azzellini, G. C.; Balzani, V.; De Cola, L.; Vögtle, F. J. Am. Chem. Soc. 1998, 120, 12187.

12. Aida, T.; Jiang, D.-L. Nature 1997, 388, 454.

13. Bar-Haim, A.; Klafer, J. J. Am. Chem. Soc. 1997, 119, 6197.

14. Pollak, K. W.; Leon, J. W.; Fréchet, J. M. J.; Maskus, M.; Abruna, H. D. Chem. Mater. 1998, 10, 30.

15. Vinogradov, S. A.; Lo, L.-W.; Wilson, D. F. Chem. Eur. J. 1999, 5,1338 .

16. Hofkens, J.; Latterini, L.; De Belder, G.; Gensch, T.; Maus, M.; Vosch, T.; Karni, Y.; Schweitzer, G.; De Schryver, F. C.; Hermann, A.; Mullen, K. Chem. Phys. Lett. 1999, 304, 1.

17. Maruo, N.; Uchiyama, M.; Kato, T.; Arai, T.; Akisada, H.; Nishino, N. Chem. Commun. 1999, 20, 2057.

18. Nakazono, M.; Ma, L.; Zaitsu, K. Tetrahedron Lett. 2002, 43, 8185.

19. On the basis of the results, the following mechanism seems plausible for the chemiluminescent reaction.

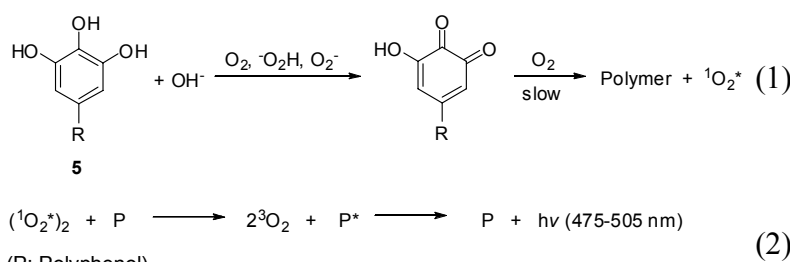

(P: Polyphenol)

During this oxidation process, an excited singlet oxygen is formed and the light emission occurs via intermolecular energy transfer as well as simple excimer emission according to reaction., ${ }^{\mathrm{a}, \mathrm{b}}$ (a) Slawinska, D.; Slawinski, J. Anal. Chem. 1975, 47, 2101. (b) Evmiridis, N. P.; Vlessidis, A. G.; Thanasoulias, N. C. Bioinorg. Chem. appl. 2007, 1. 